\title{
Power Assessment of the Human Ankle during the Stance Phase of Walking for Designing a Safe Active Prosthesis in Below-Knee Amputees
}

\author{
Sara Soltani Ch., ${ }^{1,}$, Ali Esteki \\ ${ }^{1}$ Faculty of Biomedical Engineering, Islamic Azad University, Science and Research branch, Tehran, Iran \\ ${ }^{2}$ Department of Biomechanics, Medical Physics and Engineering, School of Medicine, Shahid Beheshti University (Medical Campus), \\ Tehran, Iran
}

Email address:

sar_soltani@yahoo.com (Sara S.Ch.), aestaki@sbmu.ac.ir(Ali E.)

\section{To cite this article:}

Sara Soltani Ch., Ali Esteki. Power Assessment of the Human Ankle during the Stance Phase of Walking for Designing a Safe Active Prosthesis in Below-Knee Amputees. Applied and Computational Mathematics. Special Issue: Quality, Reliability, Safety, and Risk Modeling and Optimization. Vol. 4, No. 2-1, 2015, pp. 7-11. doi: 10.11648/j.acm.s.2015040201.12

\begin{abstract}
Many studies have been done on the biological human ankle and prosthesis replaced with this limb. Although some of these works targeted to design a prosthesis which mimics the behavior of biological ankle, additional system is required to support the elastic components; any of them have obviously introduced an active system in designing ankle prosthesis until now. In this study the power of the non-disabled human ankle joint was examined during the stance phase of walking in sagittal plane. The study aimed to better understand the ankle's dynamic behavior for designing foot prosthesis with active mechanism. Kinematic and Kinetic data of the lower limb were collected from 18 healthy, young subjects (i.e. 6 females and 13 males) walking over a speed range of slow, normal and fast (i.e. 0.86 to $1.80 \mathrm{~m} / \mathrm{s}$ ). The ankle moment versus angle curves were plotted and total, mean and maximum values of powers were calculated for each trial. The results indicated that the total, mean and maximum values of the power could increase as the walking speed increased. The main findings of this study were the total, mean and maximum values of the power as a function of the linear velocity of walking. These results showed that the speed of $1.06 \mathrm{~m} / \mathrm{s}$ was a critical velocity, below which the system was negative, showing energy consumer system, and above it the system was positive, suggesting generated energy. With the intent to design a prosthesis mimicking a biologic foot, an augmented system which is able to adjust the power to the speed is necessary.
\end{abstract}

Keywords: Natural Ankle, Active Prosthesis, Below-Knee Amputee, Moment-Angle Curves, Safety

\section{Introduction}

Many studies have been done on the biological human ankle and prosthesis replaced with this limb $[2,4,6,7,9,10$, 14]. By plotting ankle angles versus ankle moment and analyzing them in healthy males, Frigo et al. (1996) found that with an increase in walking velocity some of the loops narrowed and then changed from clockwise to counterclockwise. They claimed that such behavior was reminiscent of a mechanical system with elastic components [4]. Palmer (2002) examined the dynamics of healthy, young subjects' ankle during walking at different self-selected speeds in his MS project. He divided the characterization of the ankle into three phases of gate: controlled plantarflexion, controlled dorsiflexion, and powered plantarflexion. Palmer also modeled biological ankle as a mechanical model of spring and damper for each phase. He believed that in the third phase a torque actuator was necessary to assist the system [11]. Bateni and Olney (2002) investigated linear velocity, relative angles, net moment, power, and work at the hip, knee, and ankle joints in prosthetic gait of below-knee amputees. They showed that amputees tend to generate little power during push-off the prosthetic side [2]. In order to determine overall system characterization used in an ankle prosthesis design, Hansen et al. (2004) examined the non-disabled human ankle joint during walking. The hypothesis of the study was that the quasi-stiffness of the ankle changes when walking at different walking speeds. The hypothesis was examined using sagittal plane ankle moment versus ankle angle curves from able-bodied subjects walking over a range of speeds. They drew the variation of moment as a function of ankle angle. These data revealed that the human ankle joint could be 
effectively replaced with a rotational spring and damper for slow to normal walking speeds. However, to mimic the characteristics of the human ankle during walking at fast speeds, an augmented system would be essential [6]. Accordingly, Hansen et al. (2010) in the other study, examined the net external energy of the biologic human ankle joint and of some lower limb prosthetic ankle-foot systems during gait initiation. The purpose of this study was to better understand the ankle's behavior during the acceleration phase of walking for using in the design of improved lower limb prostheses and orthoses. They evaluated the net external energy at the ankle from some able-bodied subjects and some persons with unilateral transtibial amputations during gait initiation [7].

Although some of these works targeted to design a prosthesis which mimics the behavior of biological ankle, additional system is required to support the elastic components, any of them have obviously introduce an active system in designing ankle prosthesis until now.

All of the common prostheses use only the energy provided by the amputee himself to mimic the behavior of a healthy ankle. However, there are also possibilities to inject energy into the system using an external power source [3].

Some studies have recently been done to design active prosthesis. A research group at the Massachusetts Institute of Technology (MIT) developed the MIT Powered Foot Prosthesis by employing a high power electric actuator (150W)
[12]. In another study conduct at the Arizona State University, the SPARKy project (Spring Ankle with Regenerative Kinetics), a Robotic Tendon actuator (150W) was used to power an artificial foot. Recently, Cherelle et al. (2012)at the Vrije University Brussel, have recently concentrated their research on an active mechanism with locking and elastic components in the ankle prosthesis. They believe that adding an active system to the common prosthesis is possible and actuated devices are able to provide the necessary energy, but need heavy and bulky actuators capable of producing high torques in a short time [3].

In the present study, amount of producing or consuming energy have been assessed. The approach is introducing a pattern for designing active Prostheses.

\section{Methodology}

\subsection{Participants}

Kinematic and kinetic data were collected from 18 able-bodied subjects ( 12 males and 6 females) with an average age of 23.33 years; average mass of $66.15 \mathrm{~kg}$; and average height of $175.22 \mathrm{~cm}$. Musculoskeletal health (lack of abnormalities such Kyphosis, Scoliosis, Flat feet, Pes cavus, Flat back, Genu varum \& Knee valgus) of all subjects was confirmed (table1).

Table 1. Anthropometric characteristics of participants

\begin{tabular}{|c|c|c|c|c|c|}
\hline & Frequency & Minimum & Maximum & Mean & Standard deviation \\
\hline Age (years) & 18 & 18 & 29 & 23.33 & 3.88 \\
\hline Weight (Kg) & 18 & 45 & 88 & 66.15 & 9.68 \\
\hline Height $(\mathrm{cm})$ & 18 & 157.0 & 189.0 & 175.22 & 7.20 \\
\hline Right knee thickness $(\mathrm{cm})$ & 18 & 10.20 & 14.10 & 11.40 & 1.05 \\
\hline Left knee thickness $(\mathrm{cm})$ & 18 & 10.00 & 14.10 & 11.33 & 1.06 \\
\hline Right ankle thickness (cm) & 18 & 5.60 & 7.90 & 7.10 & 0.94 \\
\hline Left ankle thickness $(\mathrm{cm})$ & 18 & 5.50 & 7.90 & 7.12 & 0.58 \\
\hline Right leg length $(\mathrm{cm})$ & 18 & 81 & 94 & 89.56 & 3.13 \\
\hline Left leg length (cm) & 18 & 82 & 93 & 90.00 & 2.82 \\
\hline
\end{tabular}

\subsection{Experimental Protocol}

Data were collected at Ergonomics Laboratory in University of Social Welfare \& Rehabilitation Sciences by a motion an Motion Analysis system consisted of five cameras (VICON $460(100 \mathrm{~Hz})$ ) and two force plates (KISTLER (200 $\mathrm{Hz})$ ). All barefoot subjects walked on a path about 5 meters included two force plates. They walked at three different self-selected speeds (fast, normal and slow). For gathering kinematic data, 15 markers were placed on anatomical landmarks according to the Helen Hayes marker model as follows: sacrum (1 marker), ASIS (2 markers), thigh direction (2 markers), femur lateral condyle (2 markers), shank direction (2 markers), lateral malleolus (2 markers), heel (2 markers), toe (2 markers).

Before beginning each test, the subject was asked to walking with given self-selected speed (normally, fast and slowly respectively) for become familiar with the test. Each of the force plates showed ground reaction forces for only one leg separate. The subject beginning each trial with her/his preferred side (the right leg or left one). Three trials were performed at each speed, for each leg and finally 18 trials were done for each subject.

\subsection{Variable Selection and Analysis}

After determining (from force plate's vertical $\mathrm{GRF}^{1}$ data) beginning and ending points of the stance phase for each of the trials, regarding sampling frequency $(100 \mathrm{~Hz})$ ankle angle, moment and power series were drew from motion analysis system outputs. Then, ankle angular velocity data were

\footnotetext{
${ }^{1}$ Ground Reaction Force
} 
calculated from ankle angle data with numerical differentiation method.

The next step, the stance phase in each of the trials divided into three parts: Controlled plantarflexion (CP), Controlled Dorsiflexion (CD), Powered Plantarflexion (PP). The beginning of $\mathrm{CP}$ was defined as the HS or beginning of the stance phase, and the end of it was the instant when a local minimum was reached in the ankle angular position versus time curve. The second period of stance began when CP ended and its ending was coincides with first local maximum in ankle angle data. The beginning of PP was defined as when $\mathrm{CD}$ ended, and the end of PP was defined as the instant of TO (toe off). To is the instant of ending the stance phase (figure1).

The linear velocity of the trial was determined using the following equation with numerical differentiation method:

$$
V_{i}=\left(Y_{\text {sac }_{i+1}}-Y_{s a c_{i}}\right) / \Delta t
$$

Equation (1)

Where $Y_{s a c}$ is the linear position of the sacral marker at the forward direction, $i$ is the number of the data, and $t$ is the time. The mean of the $V_{i}$ values for each of the trials was regarded as linear velocity of that trial.
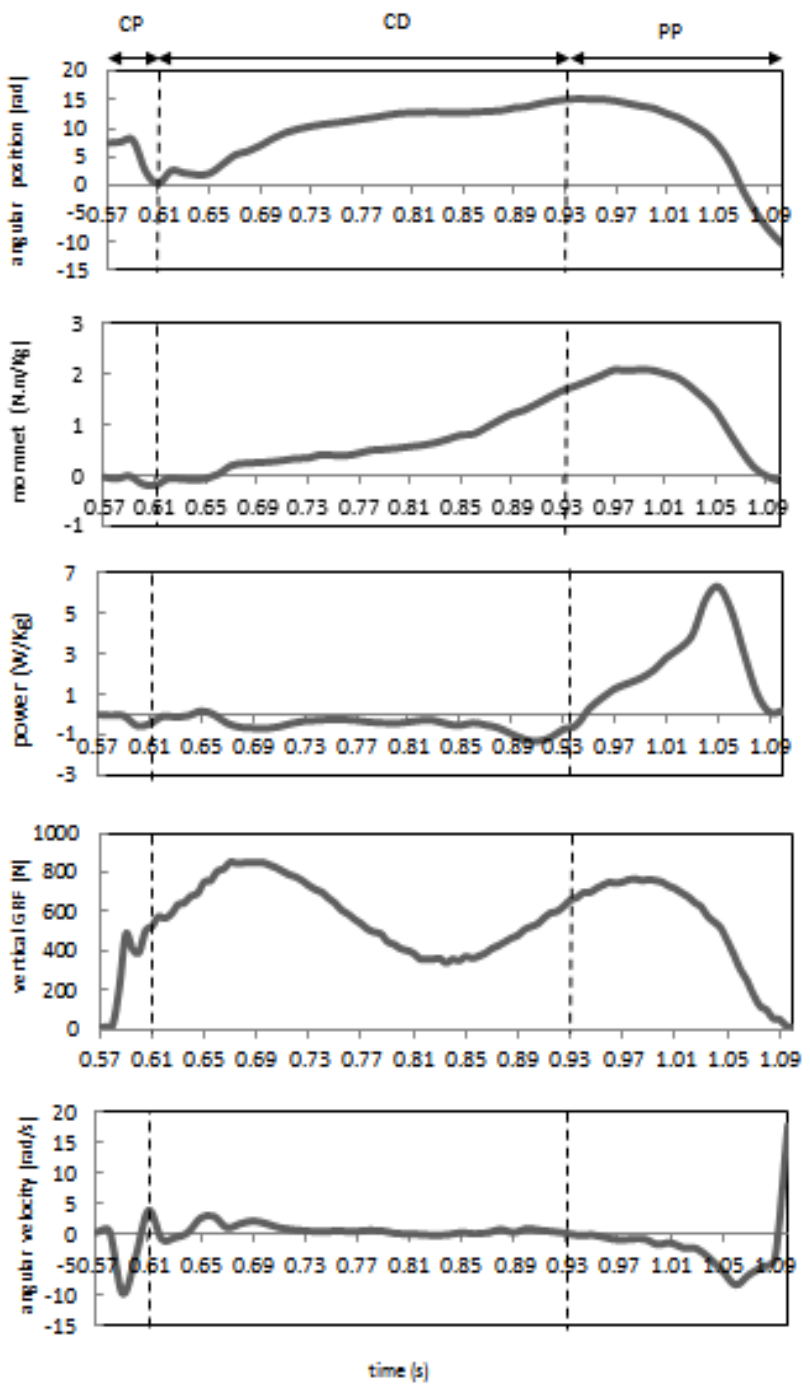

Figure 1. Kinetic and kinematic characteristics of the stance phase for a subject (velocity $=1.79 \mathrm{~m} / \mathrm{s}$ ) and the exhibition of its periods

\section{Results}

After determining the stance phase for each of the trials, total, mean and maximum power of the ankle joint was determined (figure2).

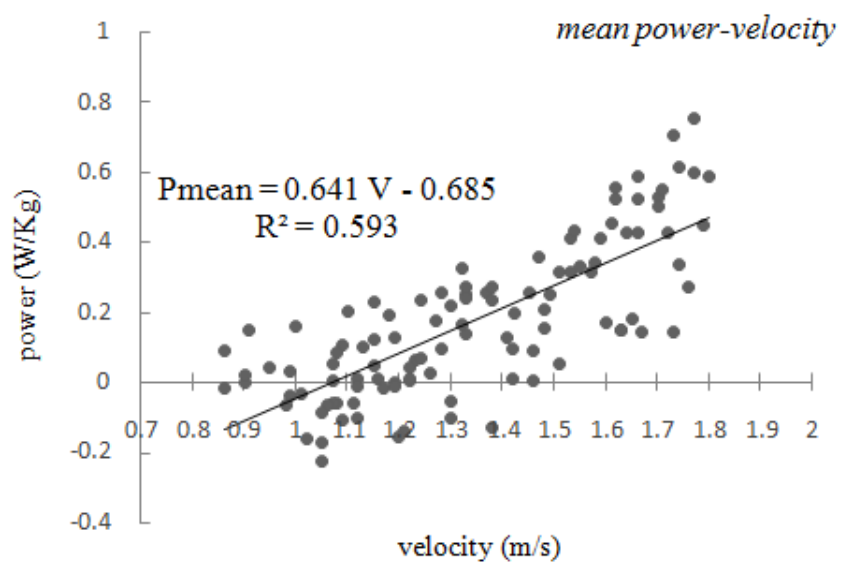

total power-velocity

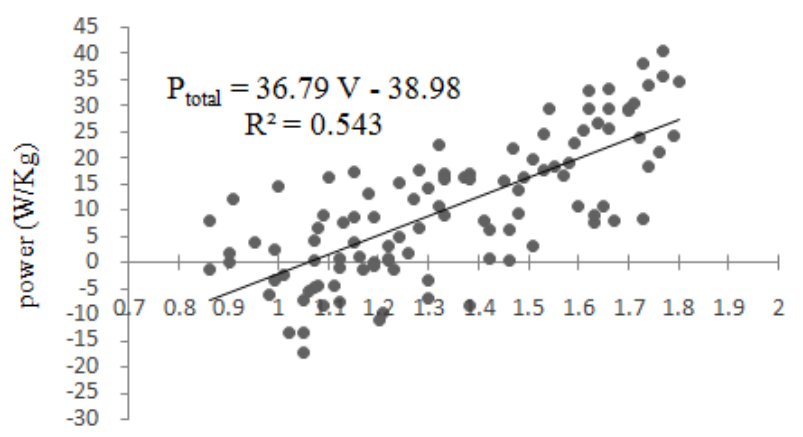

velocity $(\mathrm{m} / \mathrm{s})$

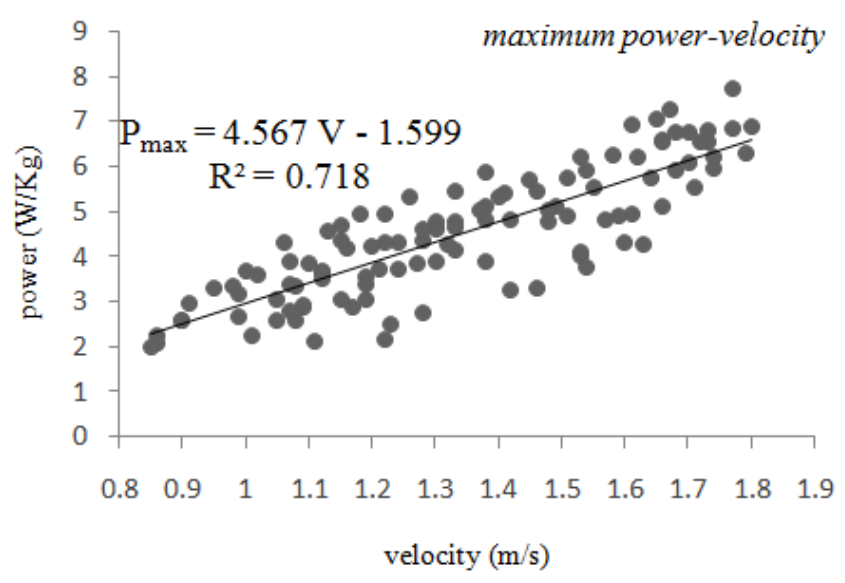

Figure 2. Mean, total and maximum ankle power curves versus linear velocity

Using regression linear indicated in equation(2), 54\% of changing in the total power values can be predicted from changing in the velocity values $(\mathrm{R} 2=0.543)$.

Total power $=36.79$ velocity -38.98

Equation (2)

Using regression linear indicated in equation(3), 59\% of changing in the mean power values can be predicted from 
changing in the velocity values $(\mathrm{R} 2=0.543)$.

$$
\text { Mean power }=0.641 \text { velocity }-0.685
$$

Using regression linear indicated in equation(4), 59\% of changing in the mean power values can be predicted from changing in the velocity values $(\mathrm{R} 2=0.543)$.

Maximum power $=4.57$ velocity $-1.60 \quad$ Equation(4)

In this study, moment versus angle curves was clockwise
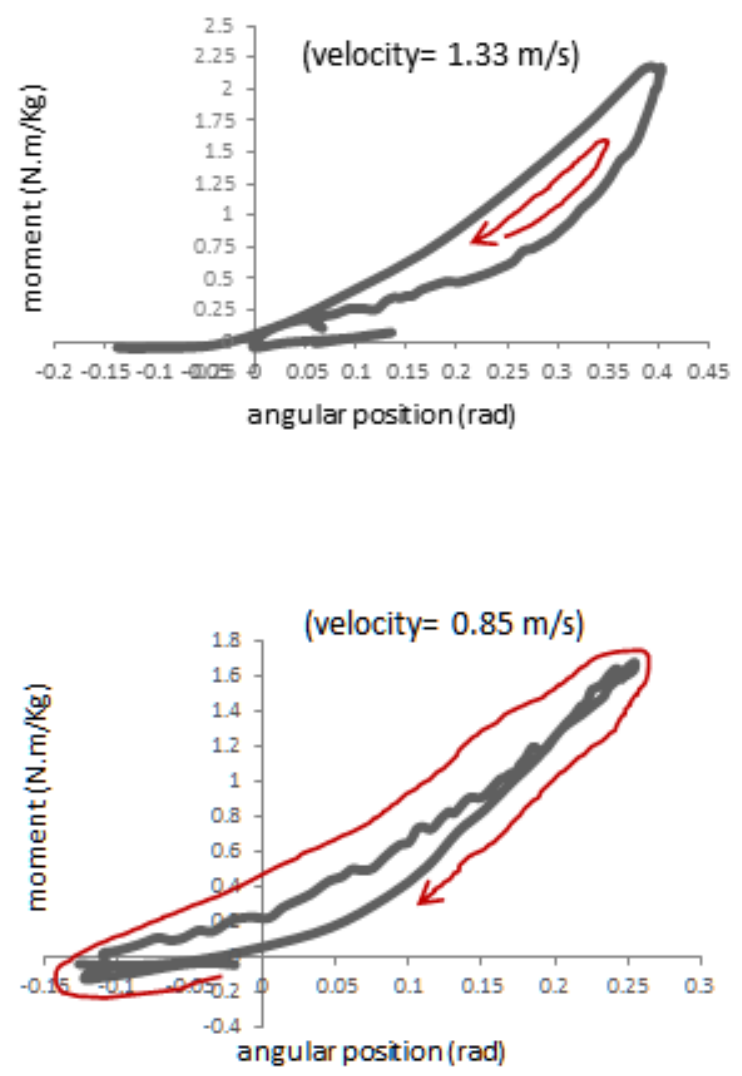

Figure 3. The moment versus ankle angle curves

\section{Discussion}

Evaluation of biological systems is common for designing a simple mechanical system which performed like the biological system. One of the most prevalent replacements for a biological limb is the ankle prosthesis used for below-knee amputees. Because of the complexity of a biological system, providing a mechanical model behaved exactly like a biological member is practically impossible (As researchers such as Hansen et al. (2004) and Bateni and Olney (2002) have proven that the prosthetic below-knee amputees using ankle prosthesis show different characteristics than healthy people in this limb). Hence, the black box method is common in assessment and simulating these limbs [2]. Thus only with considering the outputs of the at high speeds and gradually as the speed of the trial became less the area between curves would tend toward zero; when decreasing speed were continued curves became counterclockwise (figure3).

There was no regularity in the curve slope of the first stance period $(\mathrm{CP})$. In the second period of the stance phase (CD) the slope of the curves became more nonlinear as speed increased. At the last period (PP) curves were linear throughout the speed range with very good approximation.
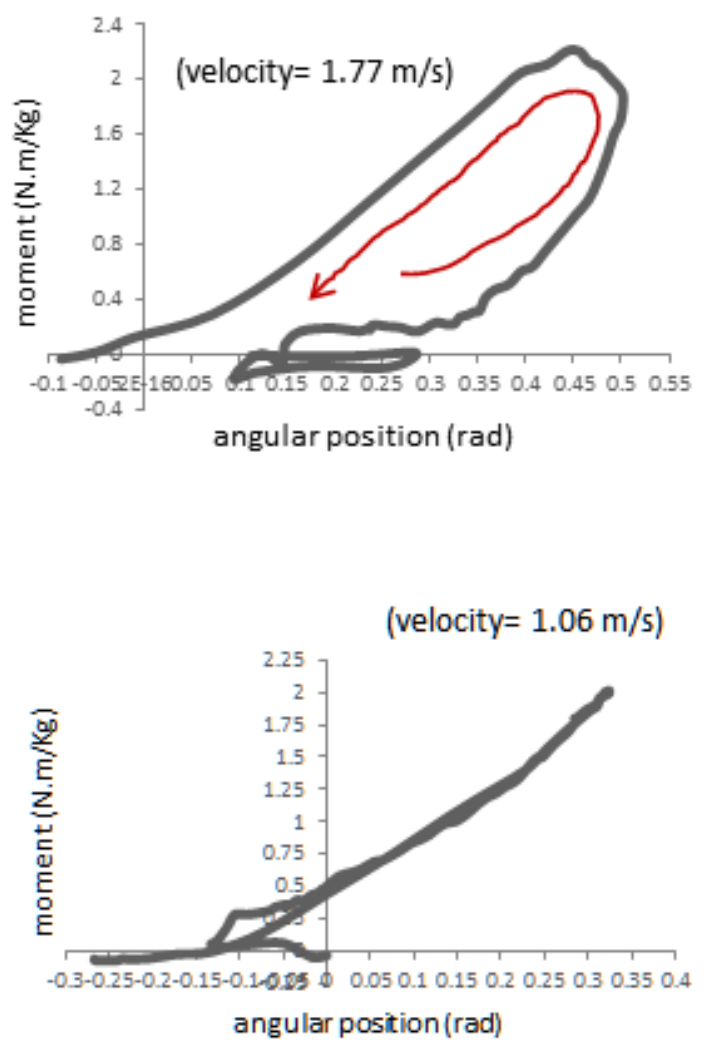

biological system, alike system is designed with the more similar outputs. As mentioned in the introduction, this study has concentrated on designing an ankle model with an active component. To reach this goal, the outputs of the power data were analyzed.

For analyzing ankle power, the moment versus ankle angle curves were evaluated. In other words, generating or consuming energy process was evaluated in each trial. The existence of the clockwise loops was confirmed energy consuming in slowly speeds. The loops were approximately disappeared in normal speeds (nearly $1.10 \mathrm{~m} / \mathrm{s}$ ) and as speeds were increased, the loops became counterclockwise; such manner is indicated energy generating in fast speeds. The area between these loops were narrower when the speed change from slow to normal until it became zero and then the area increased more and more with increased speed. How to 
change in the rotation direction and area of the loops was corresponded with results of previous researches $[4,5,6]$.

Due to the existence of high variance in the data, the first period is not discussed to study the stance phase at separated periods. Investigating the second period (CD) showed when the speed increases, the curves became more nonlinear. Also it demonstrated the power values are negative in $\mathrm{CD}$; In other words, in CD the energy is consumed. The third period (PP) was accompanied with power values being positive or energy generating.

Generally, the ankle power at the stance indicates that total, mean and maximum power increase when the speed increases. In the speed of $1.6 \mathrm{~m} / \mathrm{s}$, the mean and total power of the system became zero. Disappearing the hysteresis loops (the loops of the ankle moment versus angle curves) in this speed was confirmed the results of the ankle power versus the velocity curves. In the same way, in the slowly speeds (less than $1.06 \mathrm{~m} / \mathrm{s}$ ), total and mean power values were negative, such a result was compatible with being counterclockwise in loops. Also, for power values, being positive at speeds more than $1.6 \mathrm{~m} / \mathrm{s}$ was compatible with clockwise loops.

Up to now, no studies have quantitatively examined the relationship between total, mean and maximum ankle power and velocity. According to $\mathrm{R}$ squares of equations, results of the study is partly confirmed but producing more accurate and reliable equations needs to examine and repeat tests for more healthy subjects and with more accurate laboratory conditions.

As studies done by Palmer (2002) and Hansen et al. (2010), a mechanical system replaced with biological ankle require to an active auxiliary element $[7,11]$. These studies have not pointed to the amount of power requirements and capacity of an active element. In addition, none of the studies referred to them in the introduction has examined the amount of producing energy in this active component and none of them investigated adjusting energy in this amount of velocity.

Three equations given in this study create an appropriate pattern for these studies and researches like them, so they can evaluate the power capacity, and average and total production/consumption of the replaced systems.

\section{References}

[1] Au, S. K. and Herr, H. M., "Powered ankle-foot prosthesis," IEEE Robotics \& Automation Magazine, vol. 15, no. 3, pp. 52-59, 2008.
[2] Bateni, H., Olney, S. (2002). Kinematic and Kinetic Variations of Below-Knee Amputee Gait. Prosthet Orthotic Journal, 14, $2-10$.

[3] Cherelle, P., Matthys, A., Grosu, V., Vanderborght \& Lefeber, D. (2012). The AMP-Foot 2.0: Mimicking Intact Ankle Behavior with a Powered Transtibial Prosthesis. IEEE International Conference on Biomedical Robotics and Biomechatronics.

[4] Frigo, C., Crenna, P., Jensen, M. (1996). Moment-Angle Relationship at Lower Limb Joints during Human Walking at Different Velocities. Journal of Electromyogr. Kinesiol. Vol. 6. No. 3. PP. 177-190.

[5] Gabriel, R., Abrantes, J., Granata, K., Bulas-Cruz, J., Melo-Pinto, P., Filipe, V. (2008). Dynamic joint stiffness of the ankle during walking: Gender-related differences. Physical Therapy in Sport,9, 16-24.

[6] Hansen, A., Childress, D., Miff, S., Gard, S., Mesplay, K. (2004). The human ankle during walking: implications for design of biomimetic ankle prostheses. Journal of Biomechanics, 37, 1467-1474.

[7] Hansen, A., Miff, S., Childress, D., Gard, S., Meier, M. (2010). Net external energy of the biologic and prosthetic ankle during gait initiation. Gait \& Posture, 31, 13-17.

[8] Hitt, J., Sugar, T., Holgate, M., Bellman, R. \& Hollander, K., "Robotic transtibial prosthesis with biomechanical energy regeneration," Industrial Robot: An International Journal, vol. 36, no. 5, pp. 441-447, 2009.

[9] Houdjik, H., Doets, H., Middelkoop, M., Veeger, H.E.J. (2008). Joint stiffness of the ankle during walking after successful mobile-bearing total ankle replacement. Gait \& Posture, 27, 115-119.

[10] Moseley, A., Crosbie, J., Adams, R. (2001). Normative data for passive ankle plantarflexion-dorsiflexion flexibility. Clinical Biomechanics, 16, 514-521.

[11] Palmer, Michael Lars. (2002). "Sagittal Plane Characterization of Normal Human Ankle Function Across a Range of Walking Gait Speeds". Master's thesis. Massachusetts Institute of Thechnology.

[12] Pratt, G. A. \& Williamson, M. M., "Series elastic actuators," Proceedings of the IEEE/RSJ International Conference on Intelligent Robots and Systems, pp. 399-406, 1995.

[13] Rihs, D., \& Polizzi, I. (2001). prosthetic foot desighn. Australia: Monash Rehabilitation Thecnoloy Reasearch Unit.

[14] Shamaei, K., Cenciarini, M., Dollar, A. (2011). On the Mechanics of the Ankle in the stance Phase of the Gait. $33^{\text {rd }}$ annual conference of the IEEE EMBS. 